\title{
Optimization of the Lung Biopsy Procedure: A Primer
}

\author{
Dayananda Lingegowda $^{1}$ Bharat Gupta ${ }^{1}{ }^{\circledR}$ Anisha Gehani ${ }^{1}$ Saugata Sen ${ }^{1}$ Priya Ghosh ${ }^{1}$ \\ ${ }^{1}$ Department of Radiology, Tata Medical Center, Kolkata, West \\ Bengal, India \\ J Clin Interv Radiol ISVIR 2022;6:190-201. \\ Address for correspondence Dayananda Lingegowda, MBBS, DNB, \\ PDCC, Department of Radiology, Tata Medical Center, 14 Major \\ Arterial Road, Newtown, Kolkata, West Bengal, 700160, India \\ (e-mail: dayanandal@gmail.com).
}
Abstract
Keywords
- lung biopsy
- mediastinal biopsy
- pleural biopsy

Image-guided lung biopsy plays a very important role in the diagnosis and management of lung lesions. As a diagnostic tool, it demands a high diagnostic yield and a low complication rate. It is imperative to balance the diagnostic yield and patient safety during lung biopsies. The aim of this article is to review the standard practice guidelines of lung biopsy, to describe the techniques used to minimize the complications associated with lung biopsy, and to describe the management of complications.

\section{Introduction}

Image-guided percutaneous tissue sampling is an indispensable tool in the evaluation of pulmonary abnormalities due to its minimally invasive nature, high diagnostic accuracy, high sensitivity, and equally high specificity. Percutaneous needle biopsy of lung malignancies plays a critical role not only in the diagnosis but also in guiding and planning treatment.

\section{The Modality for Tissue Sampling}

Various methods have been described for sampling lung tissue, including percutaneous techniques, bronchoscopy, and open surgeries. ${ }^{1}$ Traditionally and to date, peripheral lung masses are sampled through percutaneous techniques. Central masses within the tracheobronchial tree are usually approached through bronchoscopy techniques and paratracheal lesions can be accessed through endobronchial ultrasound (EBUS). EBUS plays a very important role in the diagnostic staging of lung cancer. EBUS can access the anterosuperior mediastinum, posteroinferior mediastinum, and hilar nodes (2R, 2L, 3P, 4R, 4L and 7 10R, 10L, 11R, and 11L node stations). Prior to the development of EBUS, mediastinoscopy was used to sample the mediastinum. ${ }^{2}$ The diagno- sis of diffuse lung diseases requires a good volume of tissue which can be obtained through open lung biopsy or videoassisted thoracoscopic surgery. ${ }^{1}$ Mediastinoscopy plays a very important role in the nodal staging of lung malignancies. Recently, liquid biopsy is an emerging novel technique which is being used to identify new mutations in progressive diseases and can potentially substitute or even replace biopsies. $^{1}$

\section{Scope for Image-Guided Percutaneous Techniques}

All invasive procedures are associated with their own share of morbidity and mortality rates depending upon the anatomical location as well as approach (-Fig. 1). Most of the chest lesions, including chest wall, bones, pleura, peripheral lung, central lung, and mediastinal lesions can be sampled through the percutaneous approach, but with varying degrees of risks depending on the anatomical location of the lesion. Since multiple modalities are available for tissue sampling, selection of the most optimal biopsy modality should be driven by a dedicated multidisciplinary team, after carefully evaluating the procedure risk of each modality for any given anatomical location and depending upon the local availability of expertise. article published online December 17, 20221
DOI https://doi.org/ $10.1055 / \mathrm{s}-0041-1740456$. ISSN 2457-0214.
(C) 2021. Indian Society of Vascular and Interventional Radiology. All rights reserved.

This is an open access article published by Thieme under the terms of the Creative Commons Attribution-NonDerivative-NonCommercial-License, permitting copying and reproduction so long as the original work is given appropriate credit. Contents may not be used for commercial purposes, or adapted, remixed, transformed or built upon. (https://creativecommons.org/ licenses/by-nc-nd/4.0/)

Thieme Medical and Scientific Publishers Pvt. Ltd., A-12, 2nd Floor, Sector 2, Noida-201301 UP, India 


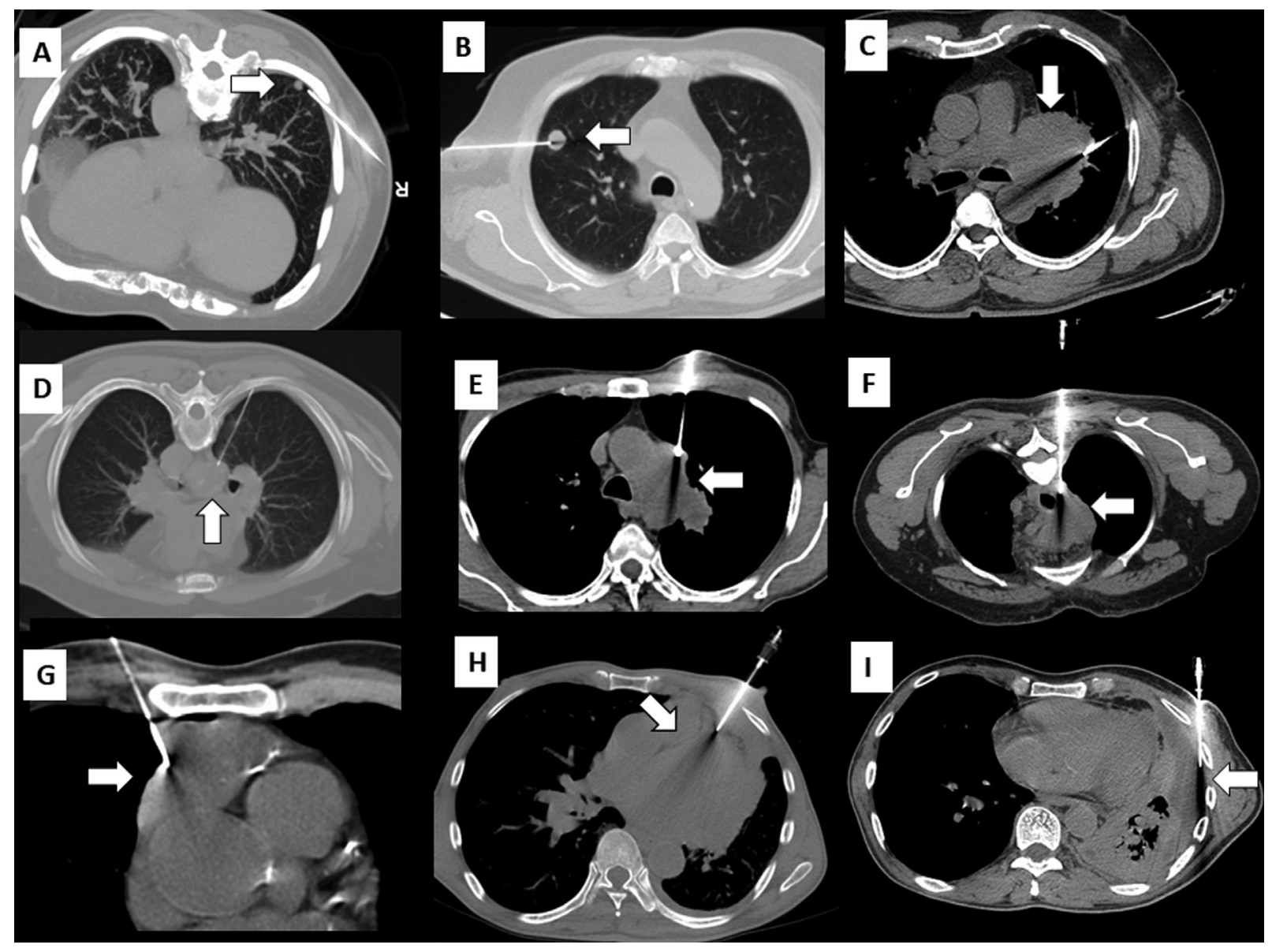

Fig. 1 Scope of image-guided biopsy. (A) Subpleural lesion biopsy. (B) Peripheral lesion biopsy. (C) Central lung mass biopsy. (D) Subcarinal node biopsy. (E) Prevascular lesion biopsy. (F) Paratracheal node biopsy. (G) Anterior mediastinal mass biopsy. (H) Pericardial mass biopsy. (J) Pleural biopsy.

\section{Preprocedure Evaluation for Lung Biopsy}

\section{Prebiopsy Imaging Evaluation}

Contrast-enhanced computed tomography (CT) or positron emission tomography CT (PET-CT) scan is desirable for patients requiring a biopsy (-Fig. 2). Contrast-enhanced $\mathrm{CT}$ is useful in identifying major vasculature in the pathway of the needle so as to avoid hemorrhagic complications during the biopsy. ${ }^{3}$ Contrast-enhanced CT/PET-CT scans are able to identify necrotic areas which need to be avoided to yield a good tissue sample and to increase the sensitivity and specificity of biopsy samples. ${ }^{3}$

\section{Anticoagulant and Antiplatelet Policy before Lung Biopsy}

All patients requiring a biopsy should have normal prothrombin time and international normalized ratio (INR) prior to the procedure. ${ }^{4}$ There is no consensus on routine assessment of platelet counts and activated partial thromboplastin time (aPTT). ${ }^{5}$ These may, however, need consideration on a case-to-case basis. Since lung biopsy carries a moderate risk of bleeding, it is recommended to stop clopidogrel 5 days before the procedure. There is no evidence to support the cessation of aspirin prior to the procedure. ${ }^{5}$ The dose of oral anticoagulants and anti-aggregatory drugs should be adjusted or stopped to reach an INR of less than 1.5 and an aPTT of not more than 1.5 times the control value. ${ }^{6}$ Platelet transfusion is recommended for a platelet count of $<50,000 / \mu \mathrm{L}$.

\section{Indications for Lung Biopsy}

Following are some of the most common indications for lung biopsy $^{1}$

1. Diagnosis of benign and malignant lung lesions.

2. Microbiologic tissue analysis in patients with known or suspected infections.

3. Staging patients with known or suspected malignancy when local spread or distant metastasis is suspected.

4. Determining the primary cell of origin in a patient with metastatic disease and an unknown primary tumor, in case of suspected malignancy.

5. Tissue analysis for biomarker, protein, or genotype analysis to subsequently guide therapy.

\section{Contraindications for Lung Biopsy}

There are no absolute contraindications to lung biopsy. ${ }^{1}$ However, there are some relative contraindications that may increase the complication rate of the procedure. The relative risks of the procedure should be weighed carefully and attempts should be made to correct or control them before the procedure. 


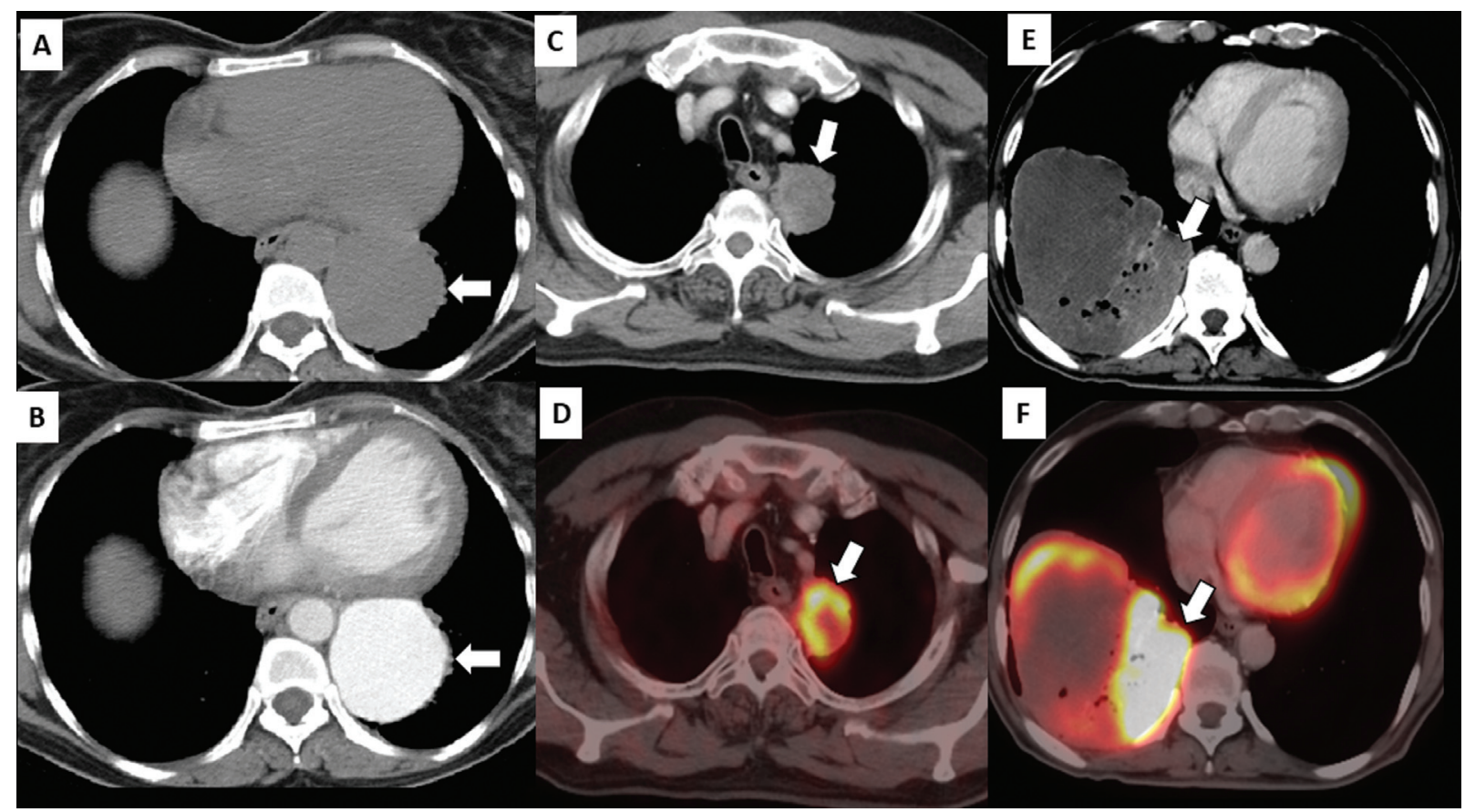

Fig. 2 Importance of contrast computed tomography (CT). (A) Case refereed for CT-guided biopsy with a detection of mass lesion on noncontrast CT (arrow). (B) Subsequently done contrast CT shows large arteriovenous malformation. (C-F) Positron emission tomography (PET) CT-guided biopsy. (C) Contrast CT shows left upper lobe lesion (arrow). (D) Corresponding PET images show eccentric activity within the lesion (arrow). (E) Another case showing large necrotic lesion (arrow). (F) PET-CT images are used to target the active area of the lesion (arrow). Teaching point: Images need to be reviewed before biopsy and targeted appropriately.

The relative contraindications for percutaneous needle biopsy include:

1. General contraindication to any interventional radiology procedure, like significant coagulopathy.

2. Severely compromised cardiopulmonary function or hemodynamic instability.

3. No safe biopsy tract or access to the lesion.

4. Uncooperative patient.

5. Uncontrolled cough.

6. Mechanical ventilation.

7. Biopsy on single functioning lung, severe chronic obstructive pulmonary disease, pulmonary hypertension, or cardiac insufficiency.

8. Suspected hydatid cyst or arteriovenous malformation.

9. Patients with forced expiratory volume in one second of less than $35 \%$ should not undergo needle biopsy without further assessment by the multidisciplinary team.

\section{Choosing the Type of Image Guidance}

$\mathrm{CT}$, fluoroscopy, and ultrasonography (US) may all be used to guide chest biopsies. ${ }^{1}$ US guidance is limited to chest wall lesions, pleural-based lesions, or peripheral lung lesions. In the vast majority of cases, $\mathrm{CT}$ is the preferred method for performing image-guided biopsies. In selected cases, during the procedure, an intravenous contrast agent can be used to differentiate target lesions from atelectasis, necrosis, and vascular structures. ${ }^{3}$ For mobile target lesions, CT-fluoroscopy guidance can be used but it increases the radiation dose to both the patient and the operator. ${ }^{3}$

\section{Fine-Needle Aspiration Cytology versus Biopsy}

Core biopsy has been shown to have higher overall sensitivity, specificity, and diagnostic accuracy. ${ }^{3}$ In view of the availability of targeted therapies for lung cancer, tissues are increasingly tested for genetic mutations. With regard to this point, a higher number of representative tissue samples need to be obtained which is generally possible only with biopsies.?

Although performing core biopsy improves the diagnostic yield of benign diagnosis, there is a slightly higher rate of complications such as pneumothorax and pulmonary hemorrhage. $^{7}$

Smaller lesions (lesions measuring $<1 \mathrm{~cm}$ ) are more likely to result in a false negative for malignancy, which may suggest an increased utility of core biopsy in such cases. $^{7}$

\section{Biopsy Needles}

Multiple different types of needles and guns are available for performing aspiration and biopsies, respectively. Some of the more commonly used fine-needle aspiration (FNA) devices include 22G Chiba needles. ${ }^{8}$ For core biopsy, semiautomatic and automatic guns are available. Generally, 18G or 20G biopsy guns will suffice (-Fig. 3 ). Using the coaxial technique helps to reduce complications and procedure time. ${ }^{8}$ Coaxial needles are much lighter in weight as compared with biopsy guns, making it easier to place, hold, and manipulate 

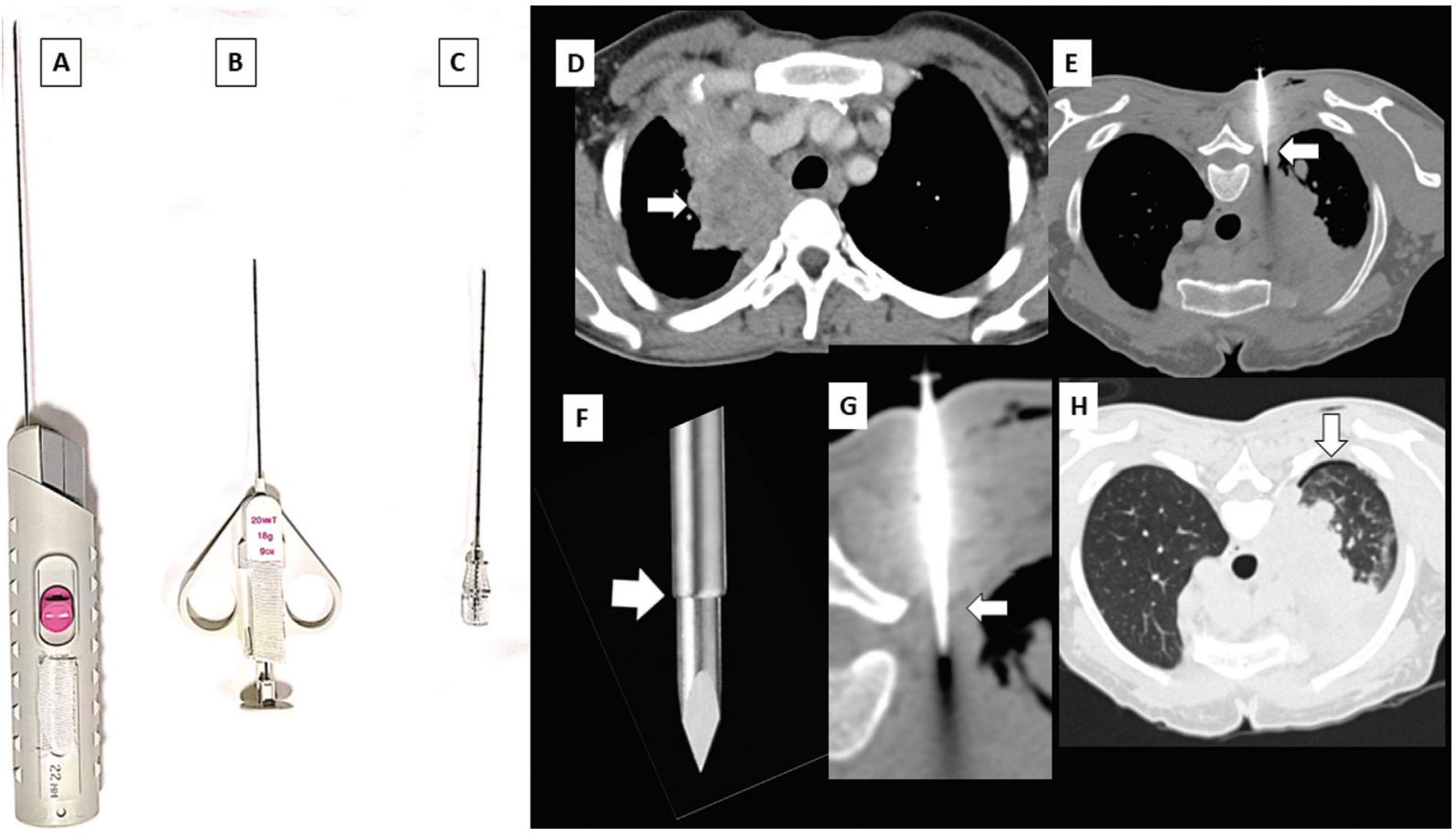

Fig. 3 Hardware for biopsy (A-C). Images showing automatic biopsy guns (A), semiautomatic guns (B), and coaxial needles (C). Importance of gap between the inner and outer part of the coaxial system (D-H). (D) Computed tomography (CT) showing large upper lobe mass infiltrating the mediastinum (arrow). (E) Lesion was approached from posterior (arrow). (F) Enlarged view of the tip of the coaxial needle demonstrating the gap between two parts of the needle (arrow). (G) Enlarged view shows the tip is inside the mass, outer part of the coaxial needle at pleura. Ideally, the entire needle needs to be inside the mass. (H) Postbiopsy images show pneumothorax due to entry of atmospheric air (ex vacuo pneumothorax). Teaching point: Adequate length of the coaxial needle is placed inside the subpleural mass to prevent pneumothorax.

during CT guidance. Also, multiple samples can be obtained through a single puncture using the coaxial technique.

In our institution, the majority of lung biopsies are done as daycare procedures. Patients are educated about all possible complications and the need for admission in case of major complications and informed consent is obtained prior to the procedure. Patients are advised to arrive for the procedure after 4 hours of fasting.

\section{Procedural Considerations}

Biopsies should be performed without sedation whenever possible. ${ }^{1}$ Sedation may be useful for patient anxiety and for elderly patients who have difficulty in lying still due to pain. A balance must be reached when using sedatives because oversedation can result in irregular respiration thus increasing the difficulty of the biopsy. ${ }^{1}$ Intravenous midazolam and fentanyl are most commonly used for sedation.

\section{Patient Positioning}

The patient should be positioned prone, supine, or lateral decubitus depending on the skin entry site chosen. Proceeding for biopsy with the patient in a seated position is generally avoided because of the potential small risk of air embolism or a vasovagal attack during the procedure. ${ }^{8}$ It is difficult for patients to maintain a consistent decubitus position and this should be avoided if possible. ${ }^{8}$

\section{Breathing Instruction}

Theoretically speaking, the breath-hold technique stabilizes the position of the diaphragm, pleural planes, lung, fissures, and ultimately the target lesion itself. In practice, breathhold capabilities can vary from patient to patient, and even the same subject may not be able to reproduce breathholding during the procedure. ${ }^{9}$ Larger tumors (greater than $2-3 \mathrm{~cm}$ ) can be targeted by free-breathing with shallow respiration. Deep breaths and coughing should be avoided during the biopsy procedure. ${ }^{9}$

If the lesion movement is very exaggerated, the patient can be instructed to maintain an inspiratory or expiratory breath hold to allow easier access to the target lesion. ${ }^{9}$ Desirable breathing techniques required during the procedure should be explained to the patient and practiced beforehand (-Fig. 4).

\section{Biopsy Technique}

It is recommended to keep the skin entry and target lesion in the same axial plane, avoiding angulation in the $z$-axis. ${ }^{1}$ In some cases where $z$-axis angulation is inevitable, gantry tilt can compensate.

Strict aseptic precautions are maintained. The skin entry site should be sterilized with a standardized antiseptic solution. The cutaneous and subcutaneous tissue is infiltrated with lidocaine (lignocaine) up to a maximum dose of $20 \mathrm{~mL}$ of a $2 \%$ solution. All attempts should be made to minimize the local anesthetic needle from coming in contact 


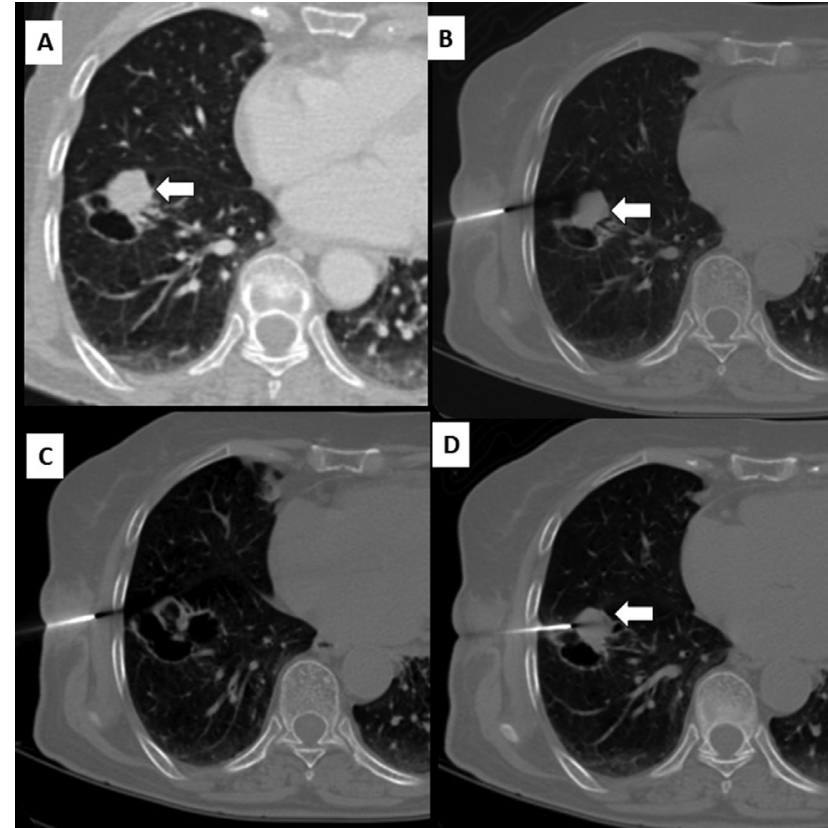

Fig. 4 Moving lesions. (A) Computed tomography (CT) showing lung cyst with the eccentric solid nodule (arrow). (B and C) Images taken during inspiration and expiration with a coaxial needle in the lateral chest wall illustrate a moving target. (D) Coaxial needle advanced into the lesion in quite breathing.

with the pleura as this is seen to increase the risk of pneumothorax even before the biopsy itself (-Fig. 5A-F).

To reduce complications, any angle correction of the coaxial needle should be done before entering into the pleura by visualizing the same on axial CT scan images. CT fluoroscopy can be used for real-time manipulation in the $x$-y-axis, particularly for difficult lesions. The disadvantage of $\mathrm{CT}$ fluoroscopy is radiation exposure to the operator.

Excessive correction should be avoided once the needle is inside the lung. When the coaxial needle is being advanced or withdrawn the patient can have free breathing or suspend respiration as per the operator's convenience. ${ }^{1}$ For lesions at the lung bases, a breath-hold in gentle expiration may make the CT images reproducible. Wherever possible, a needle entry site immediately cephalad to a rib should be chosen to avoid intercostal vessel puncture. ${ }^{1}$ Care should be taken to avoid the internal mammary vessels, major chest wall vessels, and pulmonary arteries.

Once the coaxial needle is in a satisfactory position, a biopsy gun is introduced through the coaxial needle and multiple samples are obtained. Once sufficient samples are collected, the biopsy gun and coaxial needle are removed sequentially and a pressure bandage is applied over the site.

The gantry tilting technique may be used to avoid transgressing major structures or to avoid bony structures on the path. The gantry tilt allows the craniocaudal angled approach to the lesion, yet the entire length of the needle can be seen in a single axial section. ${ }^{10}$

Postbiopsy, a noncontrast CT scan of the chest is obtained to identify immediate postprocedural complications. ${ }^{1}$

In the pediatric population, it is preferable to perform the procedure under deep sedation with local anesthesia. ${ }^{11}$ General anesthesia may be used when respiratory control is needed for lesion stability. General anesthesia allows suspending respiration at a constant point in the respiratory
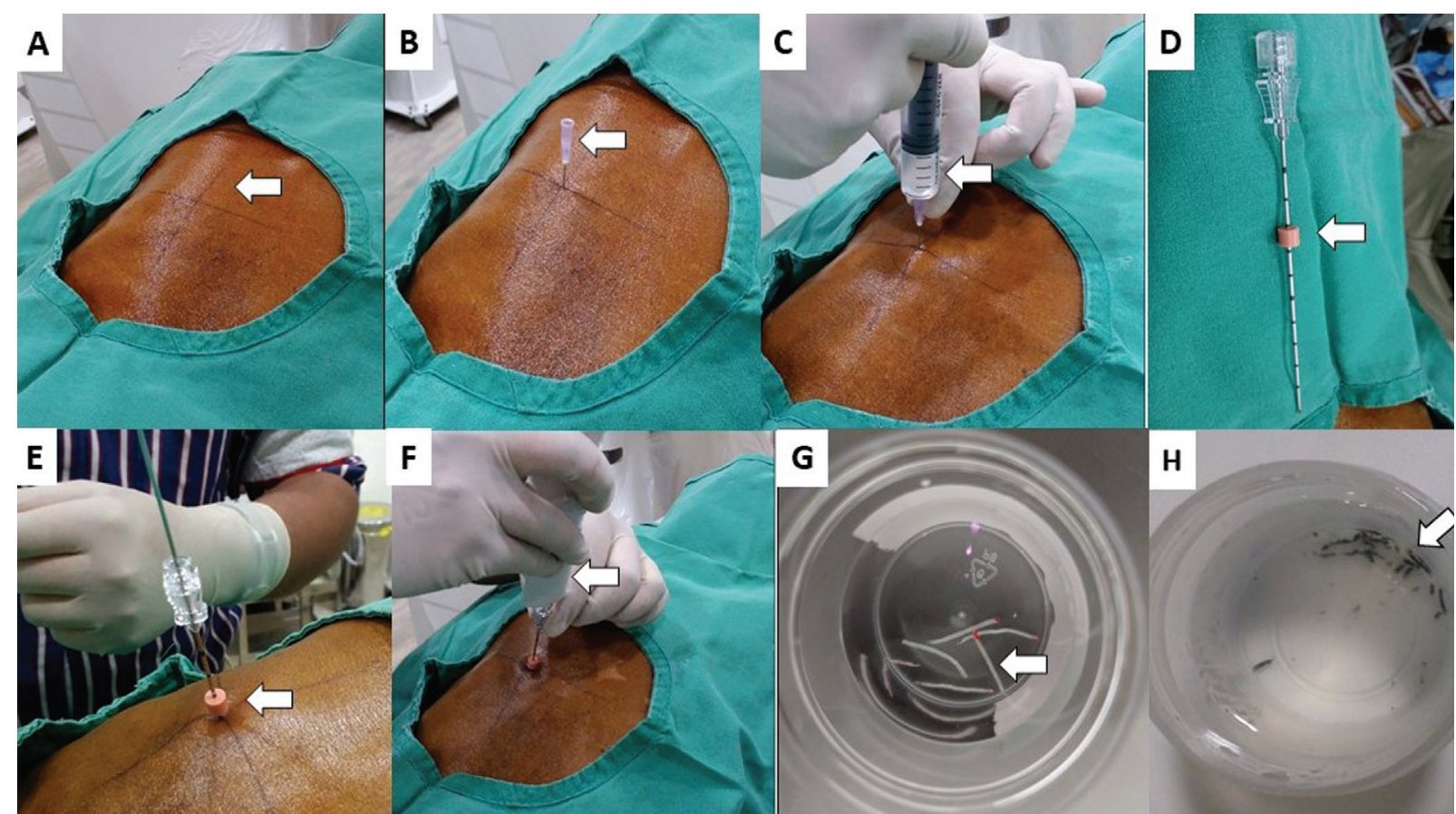

\section{G}

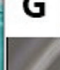

H

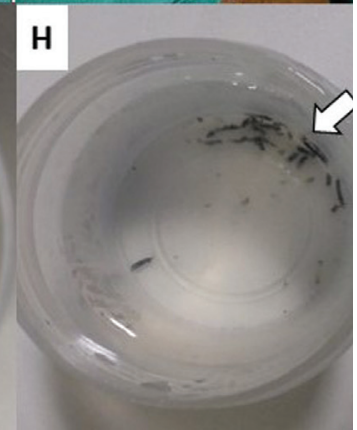

Fig. 5 Images showing steps involved in computed tomography (CT)-guided lung biopsy. (A) Surface marking (arrow). (B) CT section was taken with local anesthesia needle in place, to confirm the entry point. (C) Local anesthesia infiltration. (D) Coaxial needle with adjusted stopper (arrow). (E) Insertion of the coaxial needle. (F) Sample collection using biopsy gun. (G, H) Macroscopic appearance of samples. (G) Unfragmented sample likely to yield better results (arrow). (H) Fragmented samples may represent necrotic material (arrow). 
cycle at mid- or end-expiration and obtaining a reproducible image, especially lesions close to the diaphragm.

\section{Sample Examination at the Time of Biopsy}

For histopathological analysis and molecular testing, samples need to be collected in neutral-buffered formalin $(10 \%$ formalin). The samples which require microbiological analysis need to be separately collected and placed in the normal saline. $^{12}$

\section{Macroscopic Examination}

Visual inspection of the biopsy specimen should be performed before removing the coaxial needle, to verify the adequacy of the sample. ${ }^{1}$ There is no data elucidating the criteria for core adequacy following needle biopsy and sample sizes are known to vary among institutions. ${ }^{13}$ In our experience, a minimum of two sample cores $(2 \mathrm{~cm}$ length core each) is required. It is possible to perform touch preparation imprints on core biopsy specimens to obtain cytology. This is similar to cytological examination (-Fig. 5G, H).

\section{Immediate Pathological Examination for FNA}

There have been several reports of the value of having a cytologist or cytotechnician present at the time of the FNA cytology (FNAC) procedure. ${ }^{13}$ The immediate microscopic examination of FNAC slides reduces the negative samples.

\section{The Expected Accuracy of Sampling}

Excellent diagnostic accuracy of the sample is said to have been achieved when the false positives are reduced to less than $1 \%$ and the adequacy of the sample is over $90 \%{ }^{1}$ Sensitivity for malignancy should be within the range of 85 to $90 \%$ in lesions over $2 \mathrm{~cm}^{1}$

\section{Postbiopsy Care}

Most complications occur immediately or within the first hour of the procedure. ${ }^{1}$ Hence, patients should be kept in a supervised area for at least 1 hour or longer. Patients are observed for shortness of breath, chest pain, hemoptysis, or other symptoms.

The patients can be rolled over onto the punctured side to reduce the risk of delayed pneumothorax. ${ }^{1}$ The benefits of putting patients in the "biopsy-side down" position are questionable for management of pneumothorax. If the clinical suspicion of a pneumothorax arises, chest radiography must be obtained immediately.

\section{Management of Acute Complications}

\section{Pneumothorax}

A pneumothorax is the most common complication and is reported in up to $61 \%$ of all lung biopsies. ${ }^{1}$ Acute pneumothoraces may develop at the time of the lung biopsy procedure and immediately after the procedure. Occasionally, delayed pneumothoraces have been reported more than 24 hours after the biopsy.

\section{Management of Pneumothorax}

Where a pneumothorax is detected following a biopsy procedure, the management options include observation, aspiration, or drain insertion depending upon the symptoms and the volume of air collected within the pleural space. ${ }^{1}$ Asymptomatic small stable pneumothorax can just be observed and does not warrant any intervention. Moderate to large pneumothorax needs to be aspirated as an initial step. If postaspiration, imaging shows the persistence of pneumothorax, CT-guided small gauge (6-9F) drain placement needs to be considered. The drain must be attached to an underwater seal valve, which allows prolonged drainage for pneumothorax as well as easy outpatient management. ${ }^{1}$ In symptomatic patients, irrespective of the size of pneumothorax, one may consider a small-bore tube drain placement (-Fig. 6).

\section{Prevention of Pneumothorax}

Several techniques have been proposed to seal the path of the needle after its removal to reduce the risk of pneumothorax. One of the most common is to create a blood patch with autologous venous blood to seal the needle tract.

\section{Pulmonary Hemorrhage and Hemoptysis}

Pulmonary hemorrhage is the second most common complication of imaging-guided lung biopsy. ${ }^{8}$ Pulmonary hemorrhage may or may not be associated with hemoptysis. Minor pulmonary hemorrhage is easily identified on postbiopsy CT scan as a perilesional or needle tract ground-glass opacity. Larger pulmonary hemorrhages can be beyond the needle track. Usually, this complication does not need any treatment. One may consider placing the patient's biopsy side as the dependent position to avoid aspiration of blood into the contralateral lung. Larger symptomatic pulmonary hemorrhage needs symptomatic treatment with oxygen (-Figs. 7-9).

Most cases of hemoptysis are self-limiting requiring reassurance and supportive treatment. Aspiration of blood into the contralateral lung or massive bleeding may require the initiation of advanced cardiac life support and intubation, depending on the patient's condition.

\section{Hemothorax}

Hemothorax is extremely rare and may result from puncture of an intercostal or less commonly a large thoracic vessel, or mammary vessels in case of an anterior parasternal biopsy (- Fig. 7A-C). The timing of presentation of hemothorax after the procedure is variable and can manifest 24 hours after the procedure in $20 \%$ of cases. ${ }^{14}$ Treatment of hemothorax depends upon the clinical presentation and volume of hemothorax. A small hemothorax is treated with a longer period of observation and reassurance whereas a large volume of hemothorax may require fluid resuscitation, blood transfusion, evacuation of hemothorax using an intercostal drain, and emergent 


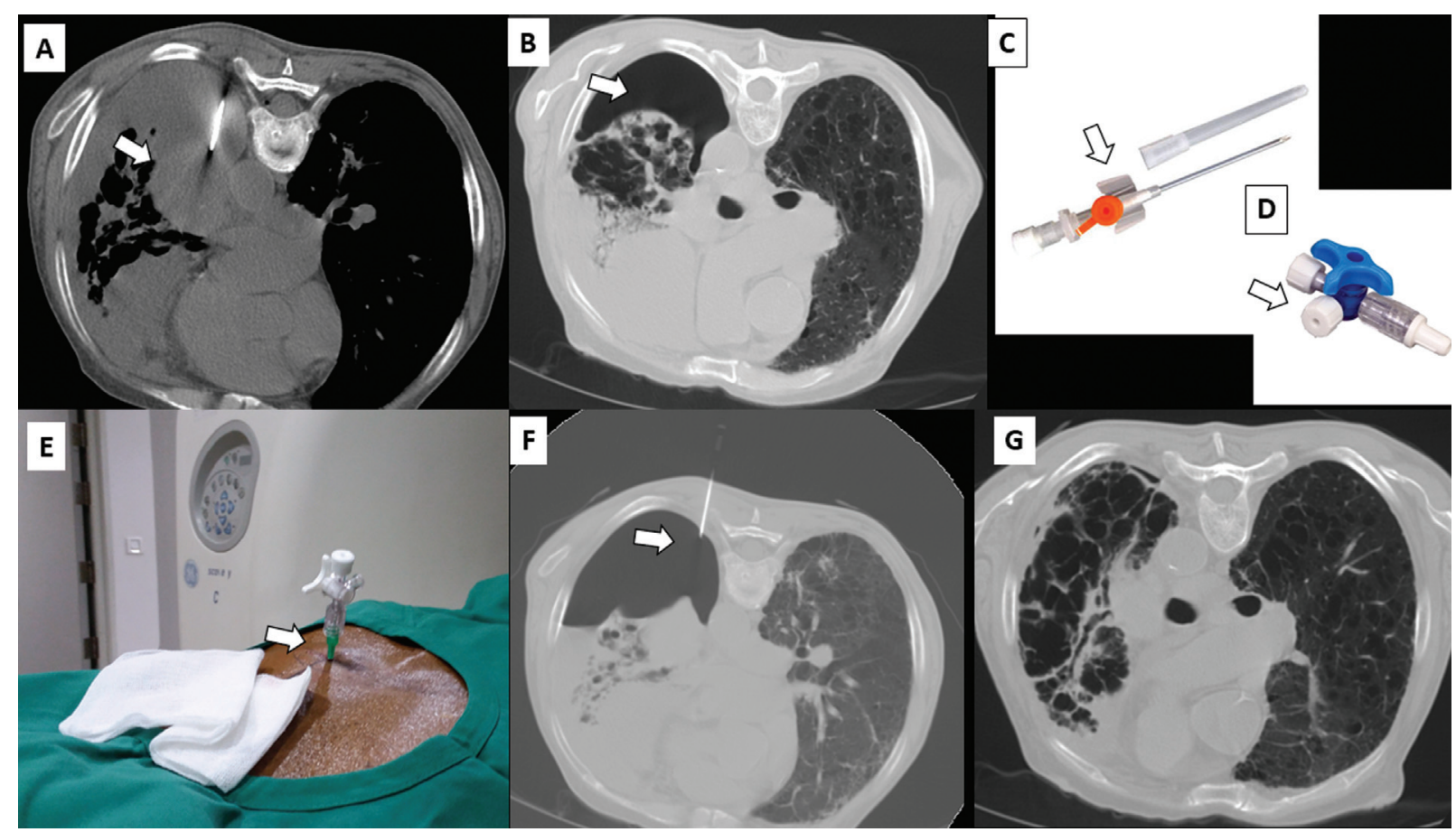

Fig. 6 Management of pneumothorax. (A) Biopsy of peripheral lung mass (arrow) in lower lobe with effusion. (B) Postbiopsy images shows pneumothorax (arrow). (C and D)18G intravenous cannula and three-way. (E) Intravenous (IV) cannula with three-way attached inserted into the pleural cavity. Aspiration of air and preexisting pleural effusion was done. (F) Postaspirated computed tomography (CT) showed satisfactory expansion of the lung.

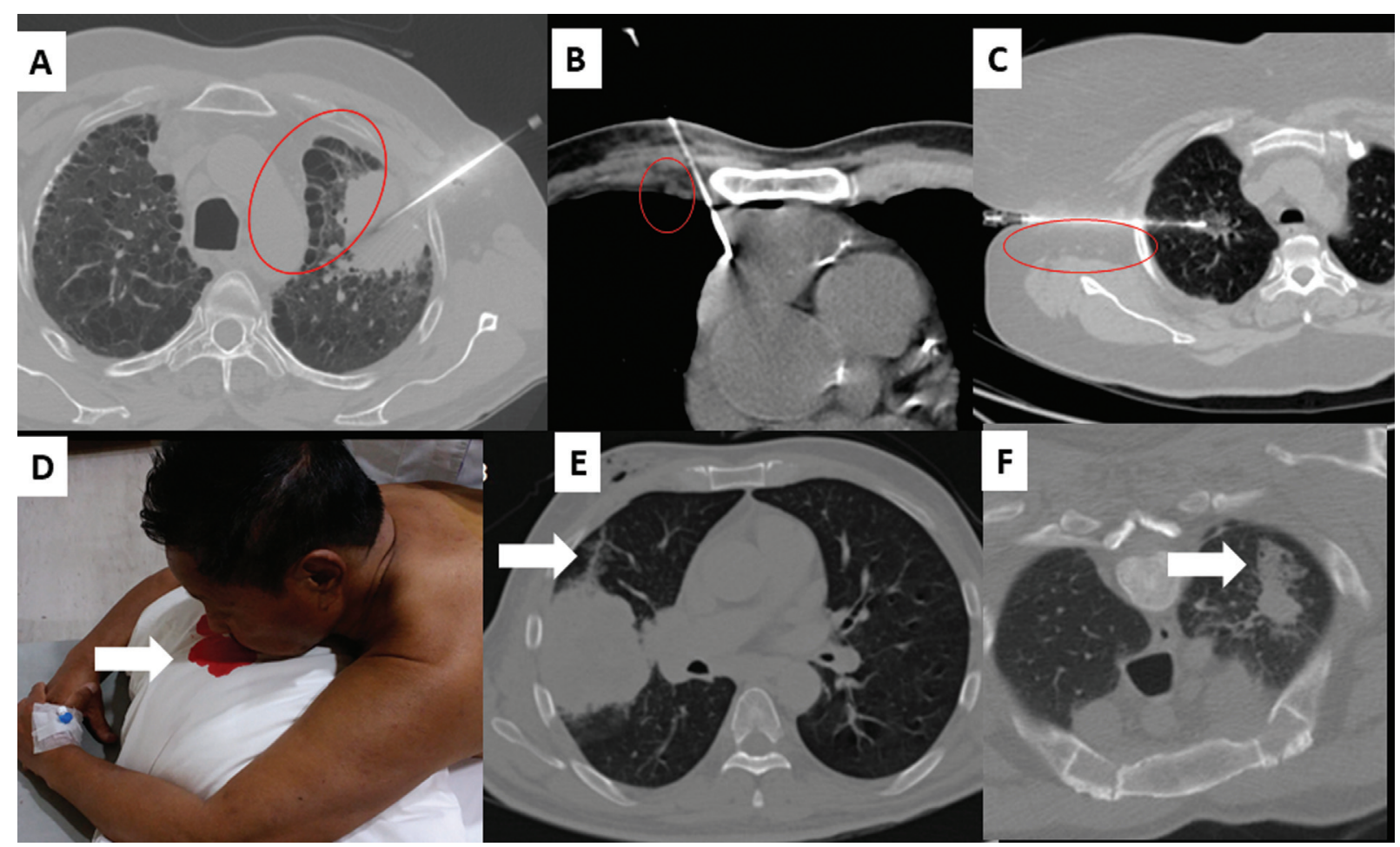

Fig. 7 Important structures to be avoided during the biopsy. (A) Biopsy of the mass lesion in the background of extensive emphysema (red circle). Needle advanced in the area, where no intervening lung parenchyma. (B) Anterior mediastinal biopsy is done avoiding internal mammary vessels (red circle). (C) Biopsy of lung lesion done avoiding lateral thoracic vessels (red circle). (D-F) Types of pulmonary hemorrhage after lung biopsy. (D) Hemoptysis after the procedure. (E) Hemorrhage along the coaxial needle track (arrow). (F) Shock wave hemorrhage (arrow). 


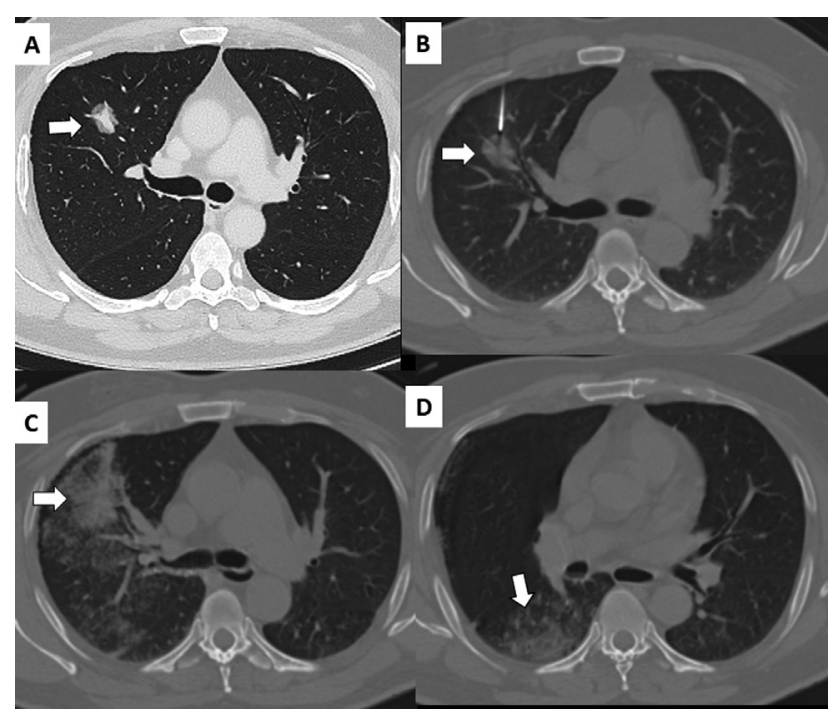

Fig. 8 Diffuse lobar hemorrhage. (A) Ground-glass lesion is seen around the right upper lobe segmental pulmonary artery (arrow). (B) Biopsy was done using 20G gun. (C and D) Postbiopsy computed tomography (CT) images show extensive alveolar hemorrhage involving the upper and lower lobe (arrow). Biopsy specimen showed adenocarcinoma of lepidic type.

surgery. Embolization of the intercostal artery is an option for persisting active extravasations.

\section{Air Embolism}

The occurrence of air embolism in the left atrium, left ventricle, or systemic circulation is rare. This can result from the placement of the needle tip in a pulmonary vein, and opening the outer cannula of a coaxial biopsy needle to the atmosphere.

The effects of air emboli are dependent on the quantity of air that has entered the circulation, whether the embolism is venous or arterial, and on the organ where they ultimately lodge. When air goes to the pulmonary vein, it can occlude any peripheral arteries, cerebral and cardiac arteries. When systemic arterial embolism is suspected, the patient should be kept in a head-down position immediately after diagnosis to prevent air from entering the cerebral vasculature. Treatment of air embolism consists of basic life support, high-flow oxygen (often with the aid of a ventilator), and intravenous fluid therapy. Hyperbaric oxygen therapy reduces bubble size by producing a diffusion gradient for oxygen into the bubble and for nitrogen out of the bubble. ${ }^{15,16}$

\section{Tumor Seeding}

Tumor seeding through the needle tract represents a very rare complication. Tumor seeding along the needle track can significantly change patient management and life expectancy.

\section{Tips and Tricks}

\section{Respect the Pleura}

Before giving local anesthesia, the distance between the skin and pleura should be measured. Care should be taken to not advance the needle tip up to the pleura, to avoid pneumothorax during the initial stage of the procedure. ${ }^{8}$
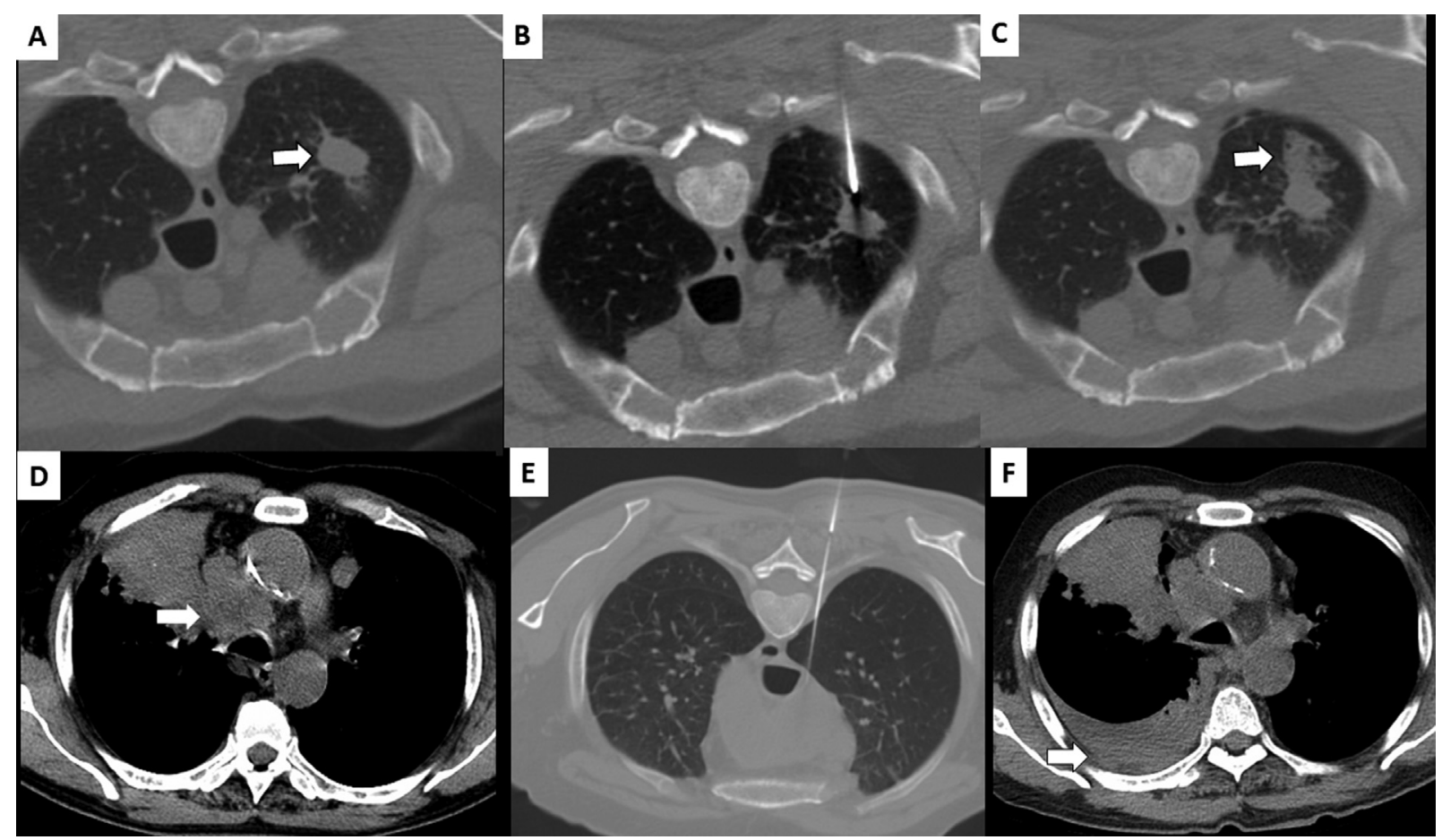

Fig. 9 Shock wave injury. (A and B) Upper lobe lesion approached from posteriorly (arrow). (C) Postbiopsy images showing a thick strip of hemorrhage along the track due to shock wave injury (arrow). During biopsy, when the biopsy gun fires, areas lateral to the needle will be also affected by the vibration. Teaching point: Major vessels should be well away from these danger zones. (D-F) Hemorrhage into pleural cavity. (D) Computed tomography (CT) scan showing right paratracheal lesion (arrow) with obstructive collapse. (E) Biopsy was done through posterior transpulmonary approach. (F) New-onset ipsilateral pleural effusion at end biopsy (arrow). 


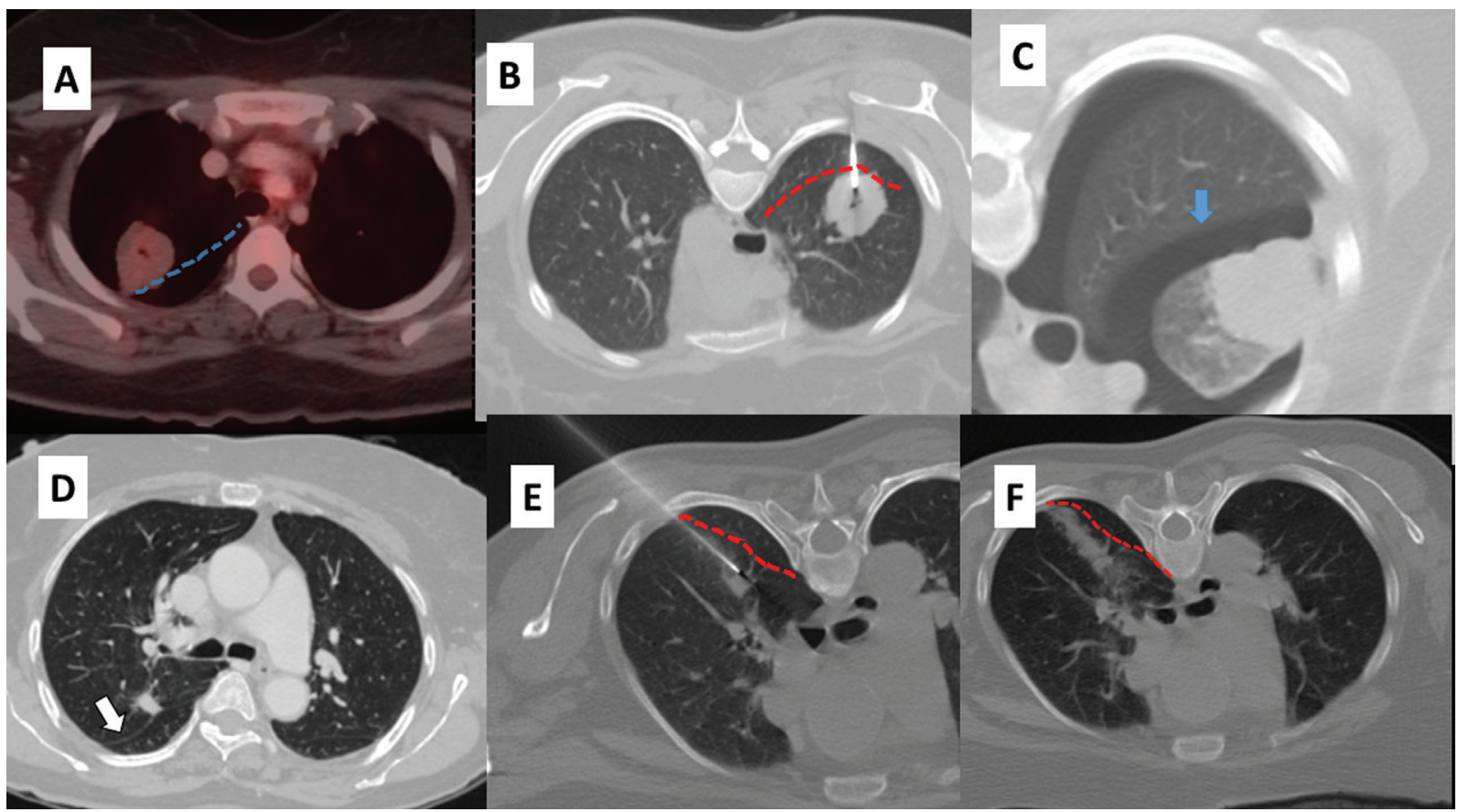

Fig. 10 Importance of avoiding fissures. (A-C) Pneumothorax due to fissural injury. (A) Positron emission tomography computed tomography (PET-CT) showing upper lobe mass anterior to major fissure (dotted blue line). (B) Biopsy was done from posterior approach crossing the major fissure (red dotted line). (C) Postbiopsy imaging showing pneumothorax outlining the fissure (arrow). (D) Another upper lobe lesion anterior to fissure (arrow). (E) Lesion was approached without crossing the fissure (dotted line). (F) Postbiopsy images shows only track hemorrhage, no pneumothorax.

As mentioned before, any angulation of coaxial needle should be done outside the pleura to minimize the number of pleural punctures which subsequently reduces the chances of repeated pleural puncture-related pneumothorax. ${ }^{8}$

\section{Respect the Fissures}

Crossing the fissures during the biopsy may increase the incidence of pneumothorax. ${ }^{9}$ The chosen needle pathway should be such that no fissures are crossed (-Fig. 10).

Avoid bullae, cysts, internal mammary artery, and lateral thoracic vessels to minimize complications any further (-Fig. 7A-C).

\section{Sterile Drape for Needle Stabilization}

In thin patients, the distance between skin and pleura is short, resulting in poor stabilization of the coaxial needle. ${ }^{9}$ Needle stabilization is also an issue during entry from the lateral chest wall, where the coaxial needle tends to fall due to gravity. In such circumstances, a sterile drape can be used as a needle stabilizer during scanning (- Fig. 11A, B).

\section{Biopsy under Pneumothorax}

Preexisting pneumothorax due to a prior procedure can be challenging. If the pneumothorax is stable and the patient is stable, then a CT-guided biopsy of lung lesion can be done. ${ }^{9}$ If the pneumothorax is progressing or symptomatic, a pigtail catheter is placed and one can proceed with the biopsy after stabilization of pneumothorax.

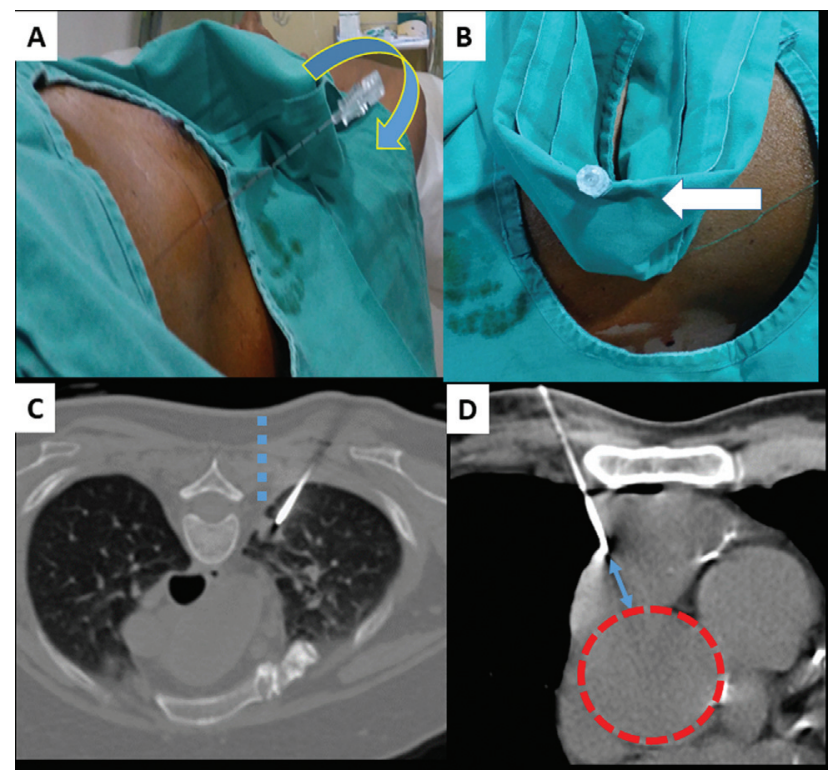

Fig. 11 (A, B) Sterile drape technique. (A) Unstable needle (curved arrow) at initial entry position due to gravitation force. (B) Needle was supported using the sterile drape (arrow) and the needle was advanced once angulations were satisfactory. (C) Approaching a subpleural lesion with a short tract (dotted line) may result in a pneumothorax lesion. Biopsy using long tract through lung parenchyma gives stability, room for manipulation, and reduces chances of pneumothorax. (D) Importance of grove length: Biopsy of the anterior mediastinal mass lying anterior to the aorta (red circle). Short grove length (blue arrow) needs to be selected to minimize the injury to vessels. 


\section{Longer Path for Subpleural Lesions}

Distance between the tips of the outer cannula and inner stylet is not the same. However, most standard needles have a 5-mm gap between the inner needle tip and the outer cannula tip. ${ }^{8}$ When performing a biopsy from a subpleural lesion, care should be taken to ensure that the outer needle tip is within the mass and not in the pleural space because that will result in pneumothorax. It is often noted that subpleural nodules are pushed away by the biopsy needle, hence a longer path transgressing some normal lung parenchyma should be chosen. If the lesion is small and subpleural, a longer path will provide better stabilization of the coaxial needle thus minimizing the chances of pneumothorax (-Fig. 11C).

\section{Biopsy Groove Length Selection}

The use of a longer groove gives better tissue yield. ${ }^{9}$ However, sometimes a short groove may be used to avoid injury to the vessels lying just anterior to the target lesion (-Fig. 11D).

\section{Hydrodissection}

The technique of hydrodissection can be used to displace the pleura along the pathway of the needle, thereby reducing the chances of pneumothorax. ${ }^{17}$ The procedure requires placing the tip of the coaxial needle in the fat plane between the vertebral body and the parietal pleura and $0.9 \%$ saline solution is then injected to displace the parietal pleura, expanding the posterior paravertebral space. Once the extrapleural space expands, the coaxial needle is advanced safely without the risk of a pneumothorax (-Fig. 12).

\section{Parenchymal Blood Patch and Pleural Blood Patching}

The intraparenchymal blood patch technique can be used to prevent pneumothorax at the time of biopsy. ${ }^{18}$ Approxi-

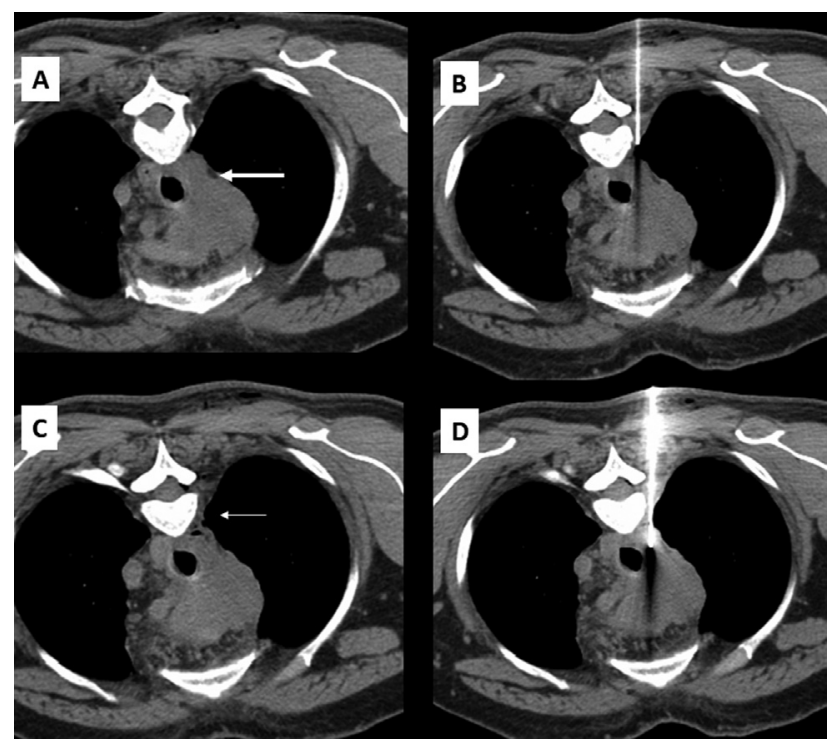

Fig. 12 Hydrodissection. (A) Computed tomography (CT) showing paratracheal node (arrow). (B) Needle in extrapleural space during hydrodissection. (C) Post-hydrodissection showing expanded extrapleural space (arrow). (D) Biopsy of the paratracheal node done without pneumothorax. mately $10 \mathrm{~mL}$ of the patient's clotted blood is placed in a 20$\mathrm{mL}$ syringe and this syringe is connected through a stopcock to a second syringe. The blood needs to be injected back and forth from one syringe to the other several times. This technique is very effective in causing fragmentation of the clotted blood. At the end of the biopsy, 4 to $8 \mathrm{~mL}$ of the fragmented blood is administered through the guiding needle as it is pulled back across each pleural surface. Blood needs to be delivered from up to $2 \mathrm{~cm}$ inside the pleural surface to $1 \mathrm{~cm}$ outside the pleural surface. The use of an autologous intraparenchymal blood patch reduces the rate of pneumothorax requiring chest tube placement (-Fig. 13A, B).

The pleural blood patching technique is used after the development of pneumothorax. ${ }^{19}$ Pneumothorax is aspirated using a cannula, and at the end of aspiration, up to $15 \mathrm{~mL}$ of autologous blood is placed into the pleural space, followed by immediate catheter withdrawal. The proposed mechanism of action of pleural blood patching is the formation of a patch of clotted blood that adheres to the site of air leakage. Pleural blood patching reduces the need for chest tube placement and hospital admission in this patient population.

\section{Intrabiopsy Manipulation}

If the coaxial needle lands at the periphery of the tumor rather than the center, it is realigned during the biopsy to get the desired trajectory. ${ }^{9}$ Final needle trajectory can be visualized on the postbiopsy images, visualized in the form of a small hemorrhage ( - Fig. 13C, D).

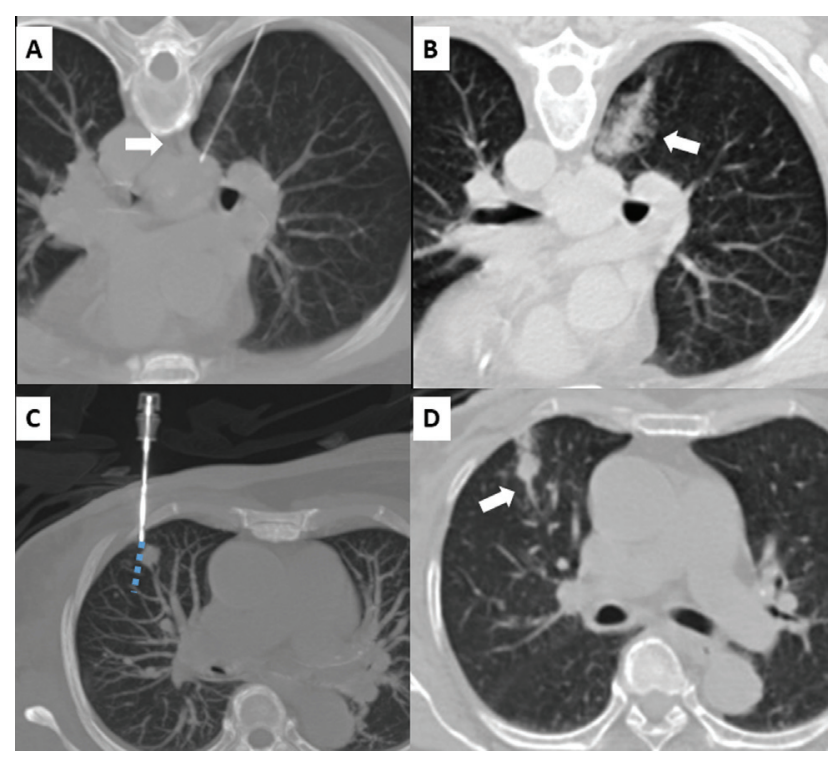

Fig. 13 (A, B) Autologous blood patch. (A) Biopsy of the paratracheal lesion through transpulmonary approach. (B) After the biopsy autologous blood patch was used to seal the track. (C, D) Final manipulation technique. (A) The coaxial needle landed in the periphery of the small lesion. Dotted line shows possible biopsy track without manipulation. Intraprocedural manipulation was done so that the biopsy track can pass through the center of the lesion. (B) Postbiopsy images confirm the final needle path (arrow). 


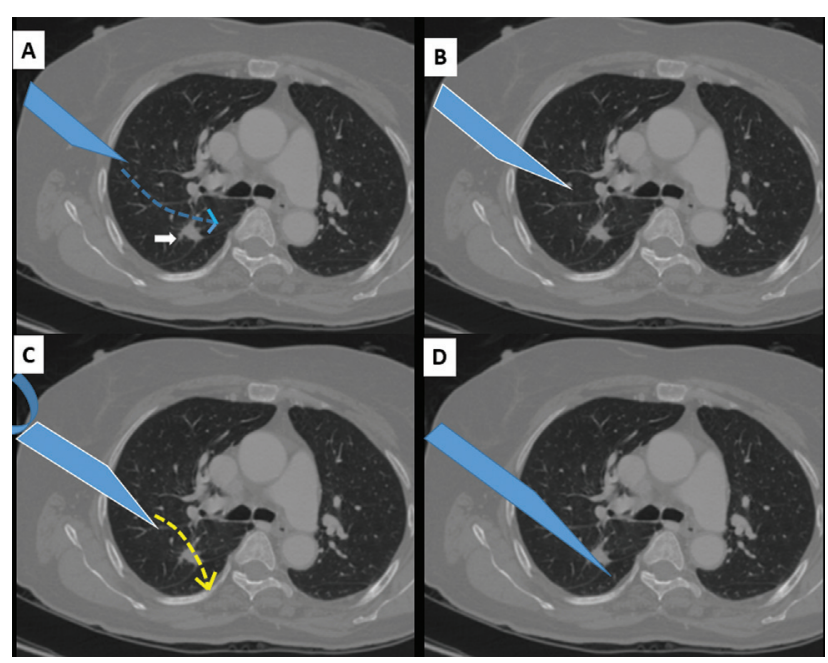

Fig. 14 Illustration of the bevel steering technique. (A) Bevel steering relies on the principle that a needle tip will drift (dotted line) toward the direction opposite that of the beveled edge. (B) If a uniform force is applied to the needle as it is partially advanced, it looks like to miss the nodule. (C) At this point, the needle is rotated 180 degrees such that the beveled edge is now facing the opposite direction. Dotted line shows change direction of drift. (D) As the needle is advanced, it will follow the new trajectory toward the nodule.

This final manipulation technique is particularly useful for the biopsy of small nodules and for those lesions located near the diaphragm or any mobile targets. ${ }^{9}$

\section{Bevel Steering technique}

If beveled needles are used as coaxial, bevel steering can be used to redirect the needle back to its ideal trajectory. ${ }^{20} \mathrm{~A}$ beveled needle has the tendency to drift in the direction away from the beveled side. By turning the bevel to face 180 degrees opposite to the intended trajectory during advancing the needle while applying a torquing pressure, the needle tip can be redirected to its intended path. If additional needle angle correction is desired, the overlying skin and soft tissues at the needle interface can be dragged during the torquing maneuver to further direct the needle tip toward the nodule (-Fig. 14).

\section{Conclusion}

CT-guided biopsy of thoracic lesions is a safe and effective way of tissue sampling. The standards practice guidelines help to reduce and manage complications.

\section{Ethical Approval}

For this type of study formal consent is not required.

Informed Consent

For this type of study informed consent is not required.

\section{Funding}

This study was not supported by any funding.

Conflict of Interest

None declared.

\section{References}

1 Manhire A, Charig M, Clelland C, et al; BTS. Guidelines for radiologically guided lung biopsy. Thorax 2003;58(11):920-936

2 Jalil BA, Yasufuku K, Khan AM. Uses, limitations, and complications of endobronchial ultrasound. Proc Bayl Univ Med Cent 2015; 28(03):325-330

3 Anzidei M, Porfiri A, Andrani F, et al. Imaging-guided chest biopsies: techniques and clinical results. Insights Imaging 2017; 8(04):419-428

4 Veltri A, Bargellini I, Giorgi L, Almeida PAMS, Akhan O. CIRSE guidelines on percutaneous needle biopsy (PNB). Cardiovasc Intervent Radiol 2017;40(10):1501-1513

5 Davidson JC, Rahim S, Hanks SE, et al. Society of Interventional Radiology consensus guidelines for the periprocedural management of thrombotic and bleeding risk in patients undergoing percutaneous image-guided interventions-part I: review of anticoagulation agents and clinical considerations: endorsed by the Canadian Association for Interventional Radiology and the Cardiovascular and Interventional Radiological Society of Europe. J Vasc Interv Radiol 2019;30(08):1155-1167

6 Patel IJ, Rahim S, Davidson JC, et al. Society of Interventional Radiology consensus guidelines for the periprocedural management of thrombotic and bleeding risk in patients undergoing percutaneous image-guided interventions-part II: recommendations [Internet]. . J Vasc Interv Radiol 2019;30:1168-84.e1

7 Schneider F, Smith MA, Lane MC, Pantanowitz L, Dacic S, Ohori NP. Adequacy of core needle biopsy specimens and fine-needle aspirates for molecular testing of lung adenocarcinomas. Am J Clin Pathol 2015;143(02):193-200, quiz 306

8 Winokur RS, Pua BB, Sullivan BW, Madoff DC. Percutaneous lung biopsy: technique, efficacy, and complications. Semin Intervent Radiol 2013;30(02):121-127

9 Tsai I-C, Tsai W-L, Chen M-C, et al. CT-guided core biopsy of lung lesions: a primer. AJR Am J Roentgenol 2009;193(05):1228-1235

10 Yueh N, Halvorsen RA Jr, Letourneau JG, Crass JR. Gantry tilt technique for CT-guided biopsy and drainage. J Comput Assist Tomogr 1989;13(01):182-184

11 Cahill AM, Baskin KM, Kaye RD, Fitz CR, Towbin RB. CT-guided percutaneous lung biopsy in children. J Vasc Interv Radiol 2004; 15(09):955-960

12 Moelans CB, ter Hoeve N, van Ginkel J-W, ten Kate FJ, van Diest PJ. Formaldehyde substitute fixatives. Analysis of macroscopy, morphologic analysis, and immunohistochemical analysis. Am J Clin Pathol 2011;136(04):548-556

13 Solomon SB, Zakowski MF, Pao W, et al. Core needle lung biopsy specimens: adequacy for EGFR and KRAS mutational analysis. AJR Am J Roentgenol 2010;194(01):266-269

14 Weinberg B, Watumull L, Landay M, Omar H. Variable presentations of thoracic biopsy related hemothorax. UJCM 2013;1(02):22-27

15 Robert M. Iatrogenic air embolism following CT-guided lung biopsy. Scotch Plains. 2012;41(04):43-44

16 Aurora T, Ward KR, Garza R, Rivers E. Iatrogenic venous air embolism. J Emerg Med 2000;18(02):255-256

17 Tyng CJ, Bitencourt AGV, Martins EBL, Pinto PNV, Chojniak R. Technical note: CT-guided paravertebral adrenal biopsy using 
hydrodissection-a safe and technically easy approach. Br J Radiol 2012;85(1015):e339-e342

18 Malone LJ, Stanfill RM, Wang H, Fahey KM, Bertino RE. Effect of intraparenchymal blood patch on rates of pneumothorax and pneumothorax requiring chest tube placement after percutaneous lung biopsy. AJR Am J Roentgenol 2013;200(06): $1238-1243$
19 Wagner JM, Hinshaw JL, Lubner MG, et al. CT-guided lung biopsies: pleural blood patching reduces the rate of chest tube placement for postbiopsy pneumothorax. AJR Am J Roentgenol 2011;197(04):783-788

20 Cham MD, Lane ME, Henschke CI, Yankelevitz DF. Lung biopsy: special techniques. Semin Respir Crit Care Med 2008;29(04): 335-349 